\title{
Research on Intelligent Control Mechanism for Wall Climbing Robot
}

\author{
Xiaomei Jiang ${ }^{1, \text { a }}$, Chaobin $\mathrm{Hu}^{1, \mathrm{~b}}$, Yannian Rui ${ }^{2, \mathrm{c}}$ \\ ${ }^{1}$ School of Mechanical Engineering, Changshu Institute of Technology, Changshu, 215500, China \\ ${ }^{2}$ College of Mechatronics Engineering, Soochow University, Suzhou, 215021, China \\ ajszjjxm@163.com, ${ }^{b}$ hcb@cslg.edu.cn, ${ }^{c}$ ryn@suda.edu.cn
}

Keywords: wall climbing robot; adaptive control; extension transformation

\begin{abstract}
Many problems of wall climbing robot on adaptive control for complex wall situation need to be solved. In this paper, based on the analysis of traditional intelligent control methods and comparison of principle of adaptive control and extension control, a new intelligent method "extension and adaptive control" was established combining adaptive control with extension control. Extension and adaptive controller was designed and preliminary verification carried out. Results show this method has better adaptability to environment than the traditional adaptive control in driven control of wall climbing robot.
\end{abstract}

\section{Introduction}

Wall climbing robot is able to move on vertical or inclined wall and complete a certain job functions. The operating environment is usually more dangerous and extreme or that humans can not adapt to an environment, such as high-rise building surface cleaning of the glass wall, outside wall spraying, ceramic tile installation, nuclear industrial inspection, weld inspection and fire emergency rescue. With the continuous advancement of intelligent control and sensor technology, using a robot to fulfill certain job tasks in such a harsh environment became a kind of human choice $[1,2]$.

Due to the complexity of the environment, a lot of problems in adaptive control of robot need to be solved [3]. Traditional adaptive control is good at dealing with constant and slow time-varying, but can not solve the unpredictable, fast time-varying problems or unexpected events in control process. For example, when wall conditions are good, adaptive driving controller can automatically adjust strategy in accordance with direction, speed to make the robot progress stably. However, when the wall situation is more complex, wall conditions is beyond the definite strategic scope, adaptive driving control can't do anything [4]. Although in recent years, intelligent control method like artificial neural networks, fuzzy control can simulate the human brain wisdom, but there is obvious lag for complex systems with unexpected events. The expert system's knowledge base and inference engine can be used to reason decision-making problems and get the relevant conclusions. However, the majority of knowledge systems and expert systems have the surface knowledge. To solve complex problems, storage, representation and processing of deep knowledge must be addressed in the knowledge-based systems to improve problem-solving capacity and flexibility [5,6].

The extension control is characterized by good performance in meeting an emergency, and uses a formal model to study the extensive possibility to expand and develop innovative rules and methods and deal with conflicts. Extension model provide a combination of qualitative and quantitative model for the artificial intelligence expression [7,8]. This paper attempts to combine extension control with adaptive control and construct extension and adaptive controller in wall climbing robot. Adaptive control is used when the environment remains unchanged or little change, and extension control when environmental change.

\section{Design of Extension and Adaptive Control System}

Basic principle of extension and adaptive control of wall climbing robot is shown in Fig.1. 


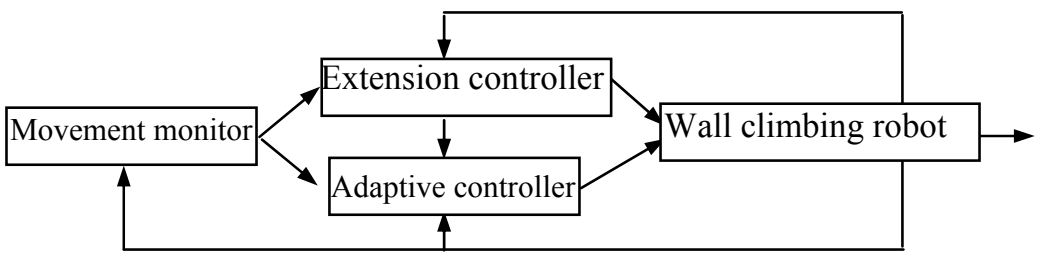

Fig. 1 Extension and adaptive control block diagram of wall climbing robot

There are three running state in control system: EC separately, EC and AC together, AC separately, monitor MM is responsible for switching between them. The structure of extension controller is shown in Fig.2. Among them general object is a equivalent including actuator, the controlled object and detection device, the remaining five modules are the key elements of extension controller.

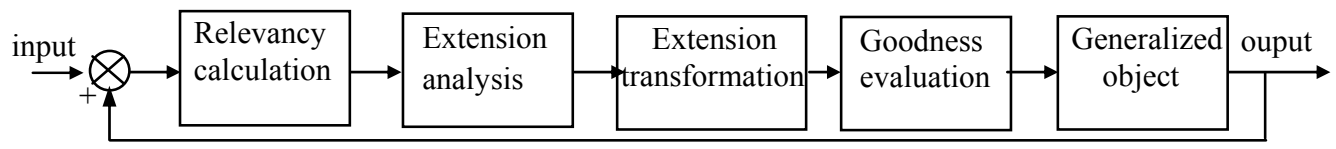

Fig. 2 The structure of extension controller

Design of Extension Controller. Establish extension model of control: the key control issues mainly contain control objectives and control conditions, and denote control objectives and control conditions as the primitive element (including the matter element, event element and relationship element)[7]. In wall climbing robot control, assumed control objectives and control conditions being:

$$
\begin{aligned}
& R=\left[\begin{array}{ccc}
\text { wall climbing robot } A, & \begin{array}{c}
\text { velocity } c_{1}, \\
\text { direction } c_{2},
\end{array} & v_{1} \\
v_{2} \\
\text { expected deviation } & c_{3}, & v_{3}
\end{array}\right]=\left[\begin{array}{ccc}
A, & c_{1} & v_{1} \\
& c_{2} & v_{2} \\
c_{3} & v_{3}
\end{array}\right] \\
& r_{1}=\left[\begin{array}{ccc}
\text { wall climbingrobot } A, & \begin{array}{c}
\text { weight } c_{4} \\
\text { slight heavy } c_{5}
\end{array} & v_{5} \\
\text { actual deviation } c_{6} & v_{6}
\end{array}\right]=\left[\begin{array}{ccc}
A, & c_{4} & v_{4} \\
c_{5} & v_{5} \\
c_{6} & v_{6}
\end{array}\right] \\
& r_{2}=\left[\begin{array}{ccc}
\text { operating conditions } B, & \text { wall conditions } c_{4} & v_{4} \\
\text { tilt degree } c_{5} & v_{5} \\
\text { outside interference } c_{6} & v_{6}
\end{array}\right]=\left[\begin{array}{ccc}
A, & c_{7} & v_{7} \\
& c_{8} & v_{8} \\
& c_{9} & v_{9}
\end{array}\right]
\end{aligned}
$$

Among them, $r_{1} \wedge r_{2}$, that is, control problem is to implement $R$ at condition $r$, denoted by: $P=R * r$. From the feature model control, then the set of characteristic state is:

$S=\left(c_{1}, c_{2}, c_{3}, c_{4}, c_{5}, c_{6}, c_{7}, c_{8}, c_{9}\right)$.

Relevancy calculation: establish extension set according to the core control issue and calculate relevancy $G(p)$ in three cases:

(1) $G(p) \geq 0$, Control is compatible, then the control can achieve the desired control objectives by the conventional adaptive control method, this phase is the adaptive control stage.

(2) $-1 \leq G(p) \leq 0$, Control is incompatible, if only the conventional adaptive control methods can not turn incompatibility into compatibility. But by adding some auxiliary means of extension control, the incompatibility control can translate into compatibility, then the conventional adaptive control can achieve desirable control, i.e. "adaptive control + extension control "stage.

(3) $G(p) \leq-1$, Control is incompatible, and adaptive control completely ineffective. This stage was divided into two cases: extension control turn incompatibility into compatibility; and opposite.

Design of Adaptive Controller. Adaptive control of robots is closely related to with dynamics. The structure of self-correction adaptive controller is shown in Fig.3. The structure of the adaptive controller based on extension set is shown in Fig.4. Consider the single-variable system, assume $x$ be set value of the system, $y$ be output of the generalized object. The system error $\delta=x-y$, error change rate $\dot{\delta}=d e / d t$. According to empirical data, determine the output variables be classical domain element and knot domain element of $\delta$ and $\dot{\delta}$. 


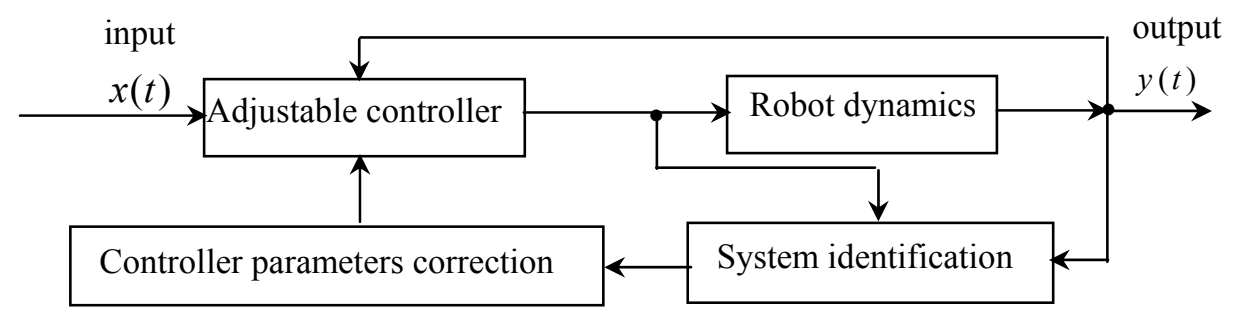

Fig. 3 Self-correction adaptive controller

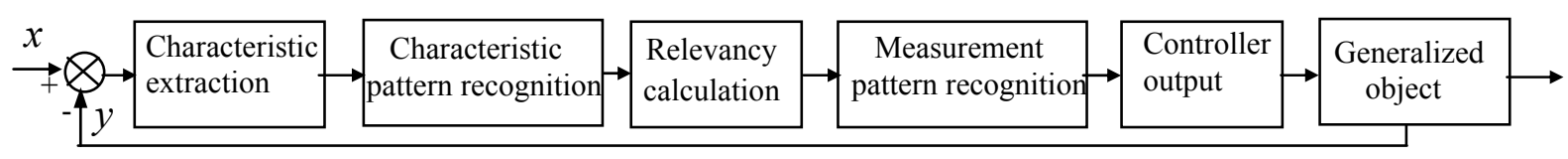

Fig. 4 The structure of adaptive extension controller

$$
\begin{aligned}
& R_{0 y}=\left[\begin{array}{lll}
y, & \delta, & \left(-\delta_{0 m}, \delta_{0 m}\right) \\
& \dot{\delta}, & \left(-\dot{\delta}_{0 m}, \dot{\delta}_{0 m}\right)
\end{array}\right] \\
& R_{y}=\left[\begin{array}{ccc}
y, & \delta, & \left(-\delta_{m}, \delta_{m}\right) \\
& \dot{\delta} & \left(-\dot{\delta}_{m}, \delta_{m}\right)
\end{array}\right]
\end{aligned}
$$

According to equation (2), establish output variables as extension set of $\delta, \dot{\delta}$, determine the division of measurement mode:

$$
\begin{aligned}
& M_{1}=\left\{S \mid G_{\bar{x}}(S) \geq 0\right\}=\left\{S \mid \delta \in\left[-\delta_{0 m}, \quad \delta_{0 m}\right], \quad \dot{\delta} \in\left[-\dot{\delta}_{0 m}, \delta_{0 m}\right]\right\} . \\
& M_{2}=\left\{S \mid G_{\bar{x}}(S) \geq 0\right\}=\left\{S \mid \delta \in\left[-\delta_{0 m}, \quad \delta_{0 m}\right], \quad \dot{\delta} \in\left[-\dot{\delta}_{0 m}, \quad \delta_{0 m}\right] \dot{\delta} \in\left[-\dot{\delta}_{m}, \delta_{m}\right]\right\}
\end{aligned}
$$

(4)

$$
\begin{aligned}
& M_{21}=\left\{S \mid a_{i-1} \leq G_{\bar{x}}(S) \leq a_{i}\right\}, i=1, \cdots, m, \text { and }-1=a_{0}<\cdots<a_{i-1} \leq a_{i} \leq \cdots \leq a_{m}=0 \\
& M_{3}=\left\{S \mid G_{\bar{x}}(S) \leq-1\right\}=\left\{S \mid \delta \notin\left[-\delta_{0 m}, \delta_{0 m}\right], \quad \dot{\delta} \notin\left[\begin{array}{ll}
-\dot{\delta}_{0 m}, & \delta_{0 m}
\end{array}\right]\right\} .
\end{aligned}
$$

Then $M_{1} \cup M_{2}$ is the controllable range of adaptive control for characteristics state $S$, the corresponding controller output calculation algorithm is as follows:

$$
\begin{aligned}
& u(t)= \begin{cases}u(t-1) & Q_{\bar{x}}(S) \geq 0 \\
-Q_{\bar{x}}(S) u_{M} \operatorname{sgn}(\delta)+\varepsilon & -1 \leq Q_{\bar{x}}(S) \leq 0 .\end{cases} \\
& \varepsilon=\left\{\begin{array}{ll}
Q_{1} \int_{0}^{t} \delta d t & |\delta| \leq e \\
& \text { others }
\end{array} .\right.
\end{aligned}
$$

Where $u_{M}>0$ is the amplitude of the controller output, $e$ is the small-scale correction, $Q_{1}$ is a constant, $e$ small positive number. Characteristics range $M_{1} \cup M_{2}$ corresponds to control problems for the compatibility problems, that is

$$
M_{1} \cup M_{2}=\{S \mid Q(P) \geq 0\} \text {. }
$$

Seen from the above, measurement pattern based on extension set only depends on classical domain and knot domain of the control characteristic without any mathematical model provided in advance and knowledge of the control system structure, so it can be well applied to control systems known inadequately.

Design of Intelligent Control Switch. In monitor intelligent control switch function is the key issues of the adaptive control system; control switch function is established based on the correlation function of extension set. Still consider the control problem divided into single-variable, assume $f$ be control characteristics, $[a, b]$ control range on $f$ of adaptive control, $[c, a]$ and $[b, d]$ warning or 
danger range on $f$ of adaptive control $([a, b] \subset[c, d])$, that is, control range of "adaptive control + extension control", establish the correlation function[8]:

$$
q(v)=\left\{\begin{array}{lr}
-\rho(v,[a, b]) & v \in[a, b] \\
\rho(v,[a, b] /[\rho[a, b]-\rho(v,[a, b])]) v=\notin[a, b]
\end{array}\right.
$$

Where, $\rho(v,[a, b])=|v-0.5(a+b)|-0.5(b-a), \rho(v,[c, d])=|v-0.5(c+d)|-0.5(d-c)$

The value of correlation function $q(v)$ has the following properties:

(1) $q(v) \geq 0$ If and only if $v \in[a, b]$; (2) $q(v) \in[-1,0]$ If and only if $v \in[a, c] \cup[b, d]$; (3) $q(v) \leq 1$ If and only if $v \notin[c, d]$; (4) $q(v)=0$ If and only if $v=a$; or $v=b$; (5) $q(v)=-1$ If and only if $v=c$; or $v=d$, For any $v \in(-\infty,+\infty)$, control switch function is defined as: $Q(P)=q(v)$

$$
Q=\left\{\begin{array}{l}
\text { adaptive Control } Q(P) \geq 0 \\
\text { extension and adaptive control } Q(P) \in(-1,0) \\
\text { extension control } \quad Q(P \leq-1)
\end{array} .\right.
$$

Based on determined range $[a, b] \subset[c, d]$ from empirical data, it is very easy to establish above control switch function, whose corresponding control form is much simpler then in artificial neural networks and fuzzy mathematics, without complicated conversion calculations and a large number of samples' statistical calculations, but also meaning is more intuitive. The objective is to improve the stability of wall climbing robot, that is:

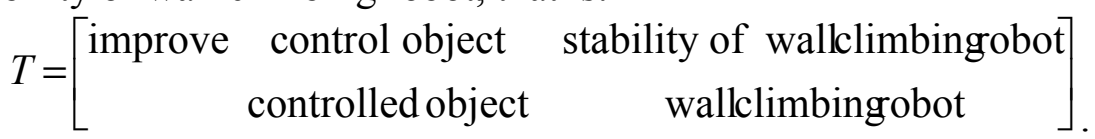

Based on extension analysis method, the following extension transformation is obtained by divergent thinking and inferiority implicit strategies of $T$ : (1) make weight center of gravity of wall climbing robot move in the opposite direction to the tilt, denoted by $H_{1}$; (2) balance the movement of wheels of wall climbing robot, denoted by $\mathrm{H}_{2}$; (3) slow down or speed up the movement speed, denoted by $\mathrm{H}_{3}$; (4) Reduce or increase the step of the span, denoted by $H_{4}$. Any of these extension transformations can make weight center of gravity transfer to improve the stability of the wall climbing robot, $H_{1} \vee H_{2} \vee H_{3} \vee H_{4} \Rightarrow T$.

\section{Conclusions}

This paper presents a new type of wall climbing robot intelligent control system design program which can combine the advantages of both adaptive control and extension control. The adaptive extension control method is formed and used in the control system of wall climbing robot, improves its level of intelligence, offers the theoretical foundation for the application of this control method.

\section{References}

[1] C.H. Liu, Y.Z. Zhao, Y. Wang: Robot Technology and Application, Vol. 2 (1996), p. 4.

[2] H. Shao, Y.Z. Zhao, Y. Wang: Manufacturing Automation, Vol. 2 (2002), p. 6.

[3] T. Zhang, C.X. Xie : Robotics (South China Technology University Press, Guangzhou 2004).

[4] Z.X. Cai: Central South University Journal (Natural Science) Vol. 5 (2005), p.721.

[5] Z.X. Cai: Intelligent control theory and application (Tsinghua University Press, Beijing 2007).

[6] D.C. Yuan: Modern Control Theory (Tsinghua University Press, Beijing 2007).

[7] L.X. Li, C.Y. Yang: Extension Strategy Generating System (Science Press, Beijing 2006).

[8] C.Y. Yang, W. Cai: Extension Engineering (Science Press, Beijing 2007). 